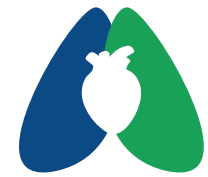

ASSOBRAFIR

C I Ê N C I A

\title{
Efeitos de um protocolo de terapia manual osteopática em pacientes submetidos à ventilação mecânica invasiva na modalidade PSV
}

\author{
Effects of a manual therapy protocol on patients undergoing \\ pressure support ventilation (PSV)
}

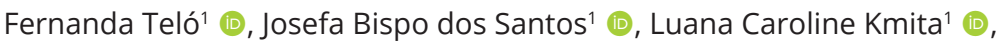
Ana Paula Rodrigues ${ }^{1}$ (D), Esperidião Elias Aquim ${ }^{1}$ (D)

\begin{abstract}
Resumo
Introdução: O ato de ventilar mecanicamente por si só acarreta alterações na mecânica respiratória, o uso da terapia manual neste cenário visa recuperar o movimento fisiológico em áreas onde existe disfunção melhorando a função dos sistemas adjacentes. Objetivo: Analisar o efeito de um protocolo de terapia manual osteopática sobre a complacência dinâmica (Cdin) e a Pressão inspiratória máxima (Pimáx) de pacientes ventilados em modo pressão de suporte (PSV). Metódos: A amostra foi composta por 12 pacientes, de ambos os sexos, com idade média de 45,9 $\pm 4,4$ anos, internados na Unidade de Terapia Intensiva (UTI) do Hospital do Trabalhador (HT) localizado na cidade de Curitiba/Paraná, no período de fevereiro a julho de 2020. O protocolo incluiu sete técnicas manuais para mobilização da caixa torácica, expansibilidade, liberação diafragmática e liberação da musculatura acessória. As variáveis analisadas foram a Cdin e a Pimáx, as medidas foram obtidas antes e imediatamente após a intervenção. Os dados com distribuição normal, avaliados pelo teste de Shapiro-Wilk, foram comparados pelo Teste t student, considerando nível de significância $p \leq 0,05$. Resultados: Observou-se diferença estatisticamente significativa nos valores de Cdin e Pimáx após utilização do protocolo, média de $81,6 \pm 12,8 \mathrm{ml} / \mathrm{cmH} 20$ $(p \leq 0,01)$ e $-41,9 \pm 4,1 \mathrm{cmH} 2 \mathrm{O}(p \leq 0,03)$ respectivamente. Conclusão: O protocolo de terapia manual proposto apresentou efeitos positivos, quanto à Cdin e Pimáx de pacientes críticos sob ventilação mecânica.
\end{abstract}

Palavras-chave: Terapia Manual, Mecânica Respiratória, Ventilação Mecânica, Fisioterapia.

1Programa de Pós-graduação em Terapia Intensiva, Faculdade Inspirar, Curitiba, PR Brasil

Como citar: Teló F, Santos JB, Kmita LC, Rodrigues AP, Aquim EE. Efeitos de um protocolo de terapia manual osteopática em pacientes submetidos à ventilação mecânica invasiva na modalidade PSV. ASSOBRAFIR Ciênc. 2021;12:e43697. https:// doi.org/10.47066/2177-9333.AC.2020.0018

Submissão em: Maio 14, 2021

Aceito em: Junho 02, 2021

Estudo realizado em: Faculdade Inspirar, Curitiba, PR, Brasil.

Aprovação ética: CAEE

28608720.5.0000.5221 das Faculdades

Integradas do Brasil, nº 4.180.764.

*Autor correspondente: Luana Caroline Kmita. E-mail: luanakmita@gmail.com

\begin{abstract}
Background: The act of mechanically ventilating causes changes in ventilatory mechanics, the use of manual therapy in this scenario aims to recover the physiological movement in areas where there is dysfunction, improving the function of adjacent systems. Aim: To analyze the effect of a manual therapy protocol on the dynamic complacency (Cdin) and Maximum Inspiratory Pressure (Pimáx) of patients ventilated in pressure support mode (PSV). Methods: The sample consisted of 12 patients, of both genders, average age of $45.9 \pm 4.4$ years, admitted to the Intensive Care Unit (ICU) of the Hospital do Trabalhador (HT) located in Curitiba / Paraná, between February and July 2020. The protocol included seven manual techniques for rib cage mobilization, expansibility, diaphragmatic release and accessory muscle release. The variables analyzed were Cdin and Pimax, the measurements were obtained before and immediately after the intervention. The data with normal distribution, assessed by the Shapiro-Wilk test, were compared using the Student t test. A $p \leq 0.05$ was deemed statistically significant. Results: There was a statistically significant difference in the values of Cdin and Pimax after using the protocol, mean of $81.6 \pm 12.8 \mathrm{ml} / \mathrm{cmH} 2 \mathrm{O}$ $(p \leq 0.01)$ and $-41.9 \pm 4.1 \mathrm{cmH} 2 \mathrm{O}(\mathrm{p} \leq 0.03)$ respectively. Conclusion: The proposed manual therapy protocol had positive effects, regarding Cdin and Pimáx of critically ill patients under mechanical ventilation.
\end{abstract}

Keywords: Manual Therapy, Ventilatory Mechanics, Mechanical Ventilation, Physiotherapy. 


\section{INTRODUÇÃO}

A ventilação mecânica (VM) é um recurso terapêutico utilizado em unidades de terapia intensiva (UTI), com o objetivo de manter as trocas gasosas, diminuir o trabalho muscular respiratório, reverter ou prevenir a fadiga muscular, diminuir o consumo de oxigênio entre outros. Estudos recentes demonstram que 33\% a $46 \%$ dos pacientes admitidos nestas unidades utilizam a VM em algum momento durante a internação ${ }^{1,2}$.

A ventilação por períodos prolongados está associada a vários riscos e impacto sobre morbimortalidade destes pacientes. Dentre estes, a fraqueza muscular e a disfunção diafragmática associada à VM apresentam importante papel no atraso do desmame ventilatório. Sendo que a associação da VM prolongada com os efeitos do imobilismo no leito, resultam em perda das fibras musculares acarretando significativamente na redução da força muscular respiratória e periférica³. Além de que, o uso da VM altera o gradiente pressórico (baroinversão), devido a aplicação da pressão positiva nas vias aéreas, modificando a fisiologia respiratória, uma vez que em respiração espontânea utilizamos pressão negativa pra inflar os pulmões. Esta inversão pressórica acarreta alterações de mecânica respiratória que podem interferir negativamente na condução da VM ${ }^{4}$.

A monitorização de variáveis da mecânica ventilatória, como por exemplo a complacência dinâmica (Cdin), durante a VM é de extrema importância, utilizada para avaliar a gravidade da doença, promover ajustes necessários no ventilador mecânico, analisar os efeitos de várias modalidades terapêuticas e nortear o desmame ventilatório. Estas medidas auxiliam a manter a função pulmonar em condições ideias durante o uso de VM, reduzindo a ocorrência lesão pulmonar induzida pelo ventilador mecânico (LPIV), bem como a escolha do melhor momento para descontinuação do suporte ventilatório ${ }^{5,6}$. A pressão inspiratória máxima (Pimáx) é outra medida de grande interesse e impacto em pacientes que utilizam VM, pois a fraqueza dos músculos respiratórios está associada com a necessidade de VM prolongada e a falência no desmame ventilatório ${ }^{7}$.

A terapia manual utilizada em pacientes sob VM visa incentivar a ventilação pulmonar, através da melhora da tonicidade e elasticidade dos músculos abdominais e torácicos e mobilização dos componentes da caixa ${ }^{8,9}$. O método é baseado em posicionamentos, mobilizações das articulações costovertebrais e costacondrais, alongamentos musculares, apoios manuais para aumentar a pressão intra-abdominal e manobras miofasciais ${ }^{10}$.

Estudos apontam que terapia manual tem papel importante na melhora da mobilidade e função pulmonar. Sendo que manobras osteopáticas aplicadas à caixa torácica estão associadas à redução no tempo de internamento e mortalidade em pacientes com diagnóstico de pneumonia, apresentando ainda redução nas falhas do desmame ventilatório ${ }^{11,12}$.
Muito se sabe sobre a eficácia da terapia manual osteopática na cervicalgia e lombalgia, porém a literatura ainda é escassa no que diz respeito à utilização e eficácia de tais técnicas na musculatura e função respiratória ${ }^{13}$. Diante do exposto, considerando as alterações da mecânica respiratória e disfunção dos músculos respiratórios ocasionadas pela VM, justifica-se a realização deste estudo com o objetivo de analisar o efeito de um protocolo de terapia manual osteopática na Cdin do sistema respiratório e na Pimáx de pacientes sob VM em modo pressão de suporte (PSV).

\section{MÉTODOS}

Trata-se de um ensaio clínico, realizado na cidade de Curitiba - Paraná, desenvolvido na Unidade de Terapia Intensiva (UTI) do Hospital do Trabalhador (HT), no período de fevereiro a julho de 2020. O estudo foi aprovado pelo Comitê de Ética e Pesquisa das Faculdades Integradas do Brasil, CAEE 28608720.5.0000.522, sob parecer $\mathrm{n}^{\circ} 4.180 .764$.

A amostra foi composta por 12 pacientes, de ambos os sexos, que estiveram internados na UTI do hospital no período de coleta de dados e que preencheram os critérios para inclusão do estudo.

Os critérios para inclusão no estudo foram indivíduos de ambos os sexos, com idade igual ou acima de 18 anos, submetidos à intubação orotraqueal (IOT) e ventilação mecânica invasiva > 24 horas, em modo espontâneo (pressão de suporte - PSV), acordados e colaborativos, com score $\geq 10$ na Escala de Coma de Glasgow e que concordaram em participar da pesquisa através da assinatura do Termo de Consentimento Livre e Esclarecido (TCLE). Foram excluídos do estudo os indivíduos que apresentaram instabilidade hemodinâmica (PAS $\geq 180 \mathrm{mmHg}$ ou $\leq 90 \mathrm{mmHg}, P A D \geq 110 \mathrm{mmHg}$ ou $\leq 50 \mathrm{mmHg}$, FC $\geq 120 \mathrm{bpm}$ ), fratura de arcos costais e/ou coluna vertebral, hipertensão intracraniana (HIC), uso de sedativos e quaisquer restrições para mudança de decúbito no leito.

A ficha de coleta foi elaborada pelos pesquisadores, contendo: dados pessoais, diagnóstico clínico, altura e peso, dispositivos utilizados, sinais vitais, tempo de VM e valores de Cdin e Pimáx. Os sinais vitais foram coletados antes e imediatamente após aplicação do protocolo, sendo estes: frequência cardíaca (FC), frequência respiratória (FR), saturação periférica de oxigênio (SPO2) e pressão arterial média (PAM).

A Cdin foi calculada através da fórmula: VC/Ppico - PEEP. Sendo VC: volume corrente, PEEP: pressão expiratória positiva e Ppico: pressão de pico. A força muscular diafragmática foi analisada através da medida da Pimáx, utilizando um manovacuômetro analógico, esta medida foi obtida com o paciente em posição fowler no leito, com a peça bucal adaptada na ponta do tubo orotraqueal, e então foi solicitado ao paciente que realizasse um esforço inspiratório máximo a partir de uma expiração completa, 
ou seja, desde o volume residual. Foram realizadas três medidas e foi considerado o maior valor alcançado. As medidas de Cdin e Pimáx foram obtidas antes e logo após a utilização do protocolo de terapia manual.

O protocolo utilizado constitui-se de técnicas manuais descritas no livro "Tratamento Osteopático da Caixa Torácica"m4 , e foi divido em:

1. Mobilização da caixa torácica;
a. Técnica de músculo energia para disfunções costais respiratórias em braço de bomba.
b. Técnica de músculo energia para disfunção costal expiratória.
c. Técnica de músculo energia para disfunção costal inspiratória em alça de balde.
d. Técnica de intercostal em decúbito lateral.

2. Expansibilidade da caixa torácica:
a. Técnica funcional para disfunção respiratória costal em alça de balde.
b. Técnica funcional para disfunção respiratória costal em braço de bomba.

3. Liberação diafragmática:
a. Stretching da parte lateral do hemidiafragma.
b. Técnica das fibras do diafragma.

4. Liberação da musculatura respiratória acessória:
a. Técnica de relaxamento miofascial funcional para cintura escapular.

Os dados com distribuição normal, avaliados pelo teste de Shapiro-Wilk, foram comparados utilizando o teste $T$ student para amostras dependentes (Paired Samples T-Test), utilizando o software GraphPad Prism 6. Os valores foram expressos como média \pm erro padrão da média (SEM). As análises foram realizadas com nível de significância estabelecido de $5 \%$ com valor de $p \leq 0,05$.

\section{RESULTADOS}

Participaram do estudo 12 indivíduos, a caracterização da amostra é apresentada na Tabela 1.

A Figura 1 mostra a análise da Cdin do sistema respiratório antes e após a utilização do protocolo. Observou-se aumento na média da Cdin após a utilização do protocolo de terapia manual osteopática $(p \leq 0,01)$. A média da Cdin pré foi de $60,9 \pm 7,1 \mathrm{ml} / \mathrm{cmH} 2 \mathrm{O}$ e pós foi de $81,6 \pm 12,8 \mathrm{ml} / \mathrm{cmH} 2 \mathrm{O}$.

A análise da Pimáx é demonstrada na Figura 2. Houve aumento no valor de Pimáx após a utilização do protocolo de terapia manual osteopática $(p \leq 0,03)$, demonstrando melhora da força muscular diafragmática. A média de Pimáx pré protocolo foi de $-36,8 \pm 4,9 \mathrm{cmH} 2 \mathrm{O}$ e pós protocolo foi de $-41,9 \pm 4,1 \mathrm{cmH} 2 \mathrm{O}$.

A Tabela 2 mostra a comparação dos sinais vitais (FC, $\mathrm{FR}$, PAM e $\mathrm{SpO}_{2}$ ) antes e após a utilização do protocolo. Não foram observadas diferenças estatisticamente significativas para as variáveis analisadas. Todos os participantes mantiveram os sinais vitais dentro de limites de segurança após a intervenção.

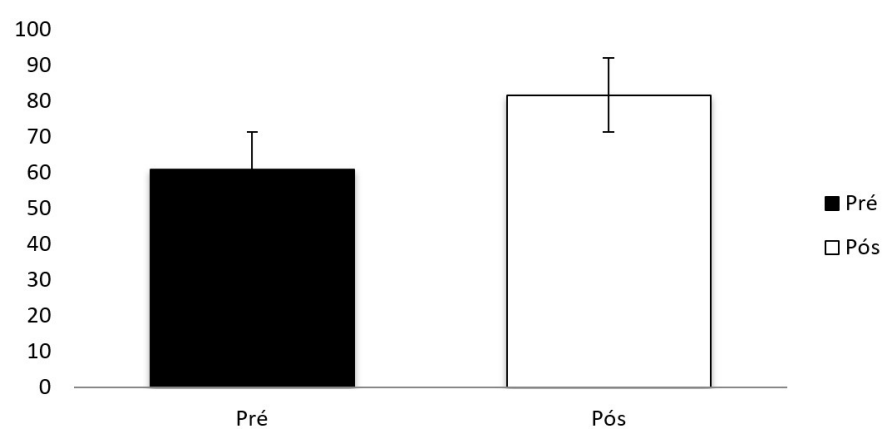

Figura 1. Análise da complacência Dinâmica pré vs pós. Cdin= complacência dinâmica. Valores foram expressos como média \pm SEM. p $\leq 0,05$. Paired Samples T-Test.

Tabela 1. Caracterização da amostra do estudo.

\begin{tabular}{|c|c|c|c|c|}
\hline Variáveis & Sexo & $\mathbf{n}$ & Média & $p$ \\
\hline \multirow[t]{2}{*}{ Idade } & Fem & 5 & $50,20 \pm 5,16$ & 0,44 \\
\hline & Mas & 7 & $42,86 \pm 6,80$ & \\
\hline \multirow[t]{2}{*}{ Altura (cm) } & Fem & 5 & $1,62 \pm 0,03$ & $0,04^{*}$ \\
\hline & Masc & 7 & $1,70 \pm 0,01$ & \\
\hline \multirow[t]{2}{*}{ Peso (kg) } & Fem & 5 & $68,00 \pm 4,21$ & 0,21 \\
\hline & Masc & 7 & $74,43 \pm 2,86$ & \\
\hline \multirow[t]{2}{*}{ IMC } & Fem & 5 & $25,90 \pm 1,79$ & 0,85 \\
\hline & Masc & 7 & $25,56 \pm 0,96$ & \\
\hline \multirow[t]{2}{*}{ Tempo em VM (h) } & Fem & 5 & $76,80 \pm 13,99$ & 0,89 \\
\hline & Masc & 7 & $79,43 \pm 12,48$ & \\
\hline
\end{tabular}

n= número amostral; $\mathrm{cm}=$ centímetros; $\mathrm{Kg}=$ kilos; $\mathrm{h}=$ horas. Valores expressos em média \pm SEM. *p $\leq 0,05$. IMC: Índice de Massa Corporal. 
Tabela 2. Comparação dos sinais vitais pré e pós protocolo.

\begin{tabular}{cccc}
\hline Variáveis & $\begin{array}{c}\text { Média } \\
\text { Pré }\end{array}$ & $\begin{array}{c}\text { Média } \\
\text { Pós }\end{array}$ & $\boldsymbol{p}$ \\
\hline Frequência Cardíaca (FC) & $94,5 \pm 6,0$ & $95,8 \pm 4,6$ & 0,6 \\
Frequência Respiratória (FR) & $17,5 \pm 1,2$ & $17,9 \pm 1,7$ & 0,6 \\
Pressão Arterial Média (PAM) & $99,9 \pm 7,1$ & $98,5 \pm 3,7$ & 0,8 \\
Saturação Periférica de $\mathrm{O}_{2}\left(\mathrm{SpO}_{2}\right)$ & $95,6 \pm 0,6$ & $96,5 \pm 0,6$ & 0,3 \\
\hline
\end{tabular}

Valores expressos em média \pm SEM.

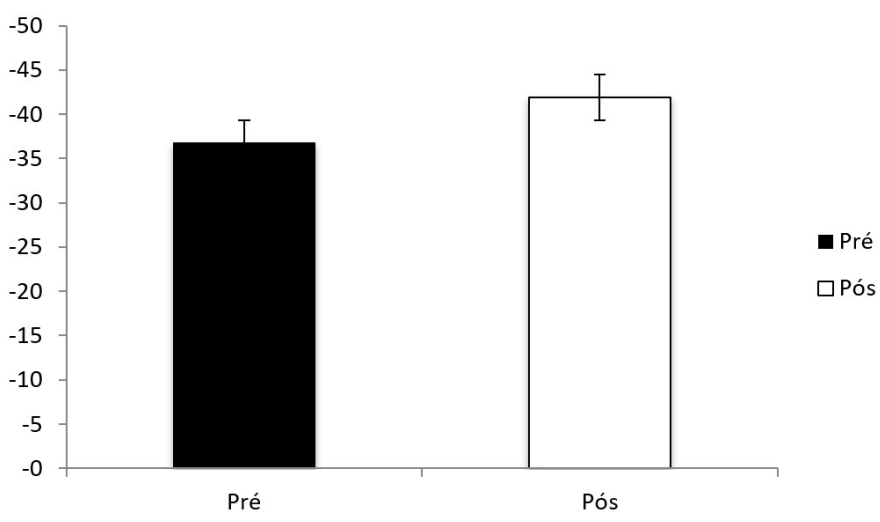

Figura 2. Análise da Pressão inspiratória máxima pré vc pós. Pimáx = pressão inspiratória máxima. Valores foram expressos como média \pm SEM. $\mathrm{p} \leq 0,05$. Paired Samples T-Test.

\section{DISCUSSÃO}

O protocolo de terapia manual osteopática apresentou efeito positivo nas variáveis analisadas, proporcionando melhora na mobilidade da caixa torácica e aumento da força muscular diafragmática, além de demonstrar segurança para sua utilização em pacientes críticos, visto que não houve alterações de sinais vitais após a intervenção nem perda dos dispositivos utilizados pelos pacientes.

Estudos demonstram que a terapia manual atua na melhora da função muscular através da flexibilização do tecido conjuntivo, com movimentos fisiológicos e acessórios das estruturas, fazendo com que uma espécie de equilíbrio neuromuscular possa acontecer ${ }^{13}$. As alterações que ocorrem na mecânica respiratória são causadas por encurtamento excessivo da musculatura inspiratória e as principais causas desse encurtamento são: alterações neuropsíquicas como (estresse), postura inadequada, patologias respiratórias, fraqueza muscular e envelhecimento.

O alongamento é utilizado com a finalidade de aumentar a flexibilidade sobre um conjunto na tentativa de promover melhora da performance, e a perda de mobilidade limita progressivamente o papel dos sarcômeros. Isso explica que, com o alongamento dos músculos respiratórios aumenta-se a mobilidade da caixa torácica, promovendo consequentemente melhora da função pulmonar ${ }^{15,16}$.
A mobilidade torácica está relacionada à integridade da musculatura respiratória, pois a mesma auxilia na expansão e retração da caixa torácica. Seu comprometimento e complicações estão ligados ao tempo de permanência do paciente em VM e imobilização no leito, as mobilizações terapêuticas atuam beneficiando a função cardíaca e pulmonar, reduzido o trabalho respiratório, proporcionando aumento dos volumes pulmonares, além de retardarem a redução do trofismo e força muscular ${ }^{13}$.

Estudos demonstram que há aumento da mobilidade torácica durante a aplicação de técnicas osteopáticas, tendo em vista que o desequilíbrio na mecânica respiratória é geralmente decorrente de encurtamento excessivo da musculatura inspiratória, resultando também na alteração da força muscular9,17.

Como observado neste estudo, houve aumento na média da Cdin após a utilização do protocolo, considerando que a Cdin engloba as propriedades elásticas e resistivas do sistema respiratório, bem como a movimentação da caixa torácica. Podemos afirmar então, que as manobras osteopáticas que melhoram a mobilidade da caixa torácica, promovendo seu arco de movimento normal, impactam diretamente e positivamente a complacência dinâmica do sistema respiratório.

$\mathrm{Na}$ fisiologia respiratória a redução da complacência pulmonar é influenciada por uma parede torácica rígida ou capacidade de distensão e recuo elástico alterados, assim a ventilação pulmonar envolve o movimento da parede torácica para produzir um gradiente de pressão que irá permitir o fluxo e a troca gasosa ${ }^{18}$.

Em ventilação espontânea a complacência total do sistema respiratório, incluindo parede torácica e pulmão, é de aproximadamente 100 a $200 \mathrm{~mL} / \mathrm{cmH} 2 \mathrm{O}$ e em indivíduos normais anestesiados e ventilados é de $70-80 \mathrm{~mL} / \mathrm{cmH} 2 \mathrm{O}$. Um pulmão rígido tem baixa complacência, como é o caso da Síndrome do Desconforto Respiratório Agudo (SDRA) que cursa com baixa complacência, enquanto que um pulmão altamente distensível, observado em pacientes com enfisema pulmonar possui complacência muito alta ${ }^{5,19}$

A força muscular altera-se quando a parede muscular se encontra encurtada, sendo assim, a mesma perde sua flexibilidade normal, impossibilitando o funcionamento ideal muscular. Consequentemente, o aumento de força muscular se dá em virtude do aumento do comprimento funcional do músculo através de alongamentos ${ }^{9,20}$.

Sendo assim, pode-se dizer que as técnicas de terapia manual combinadas, promovem um relaxamento das fibras do músculo diafragma, resultando em melhor tonicidade e força abdominal e consequentemente uma maior função diafragmática e aumento do sincronismo toraco-abdominal, resultando em aumento da força inspiratória ${ }^{20-22}$.

Corroborando com tais evidências, observamos um aumento na média da Pimáx após a intervenção, tal 
fato pode ser atribuído ao alongamento da musculatura respiratória promovido pelas técnicas manuais utilizadas. Sabe-se que, o alongamento de uma fibra muscular promove aumento do número de sarcômeros em série, promovendo melhor interação entre os filamentos de actina e miosina resultado em aumento da força muscular 23,24 .

Sabe-se que a mensuração da Pimáx é de suma importância para o diagnóstico da fraqueza muscular inspiratória, estudos apontam que pacientes com uma Pimáx menor que $-30 \mathrm{cmH} 2 \mathrm{O}$ apresentavam bom prognóstico com relação ao desmame da ventilação mecânica, enquanto que pacientes com PiMax maior que $-20 \mathrm{cmH} 2 \mathrm{O}$ falhavam no desmame, dessa forma promover a manutenção da força diafragmática de pacientes em VM é fundamental para o sucesso no desmame ventilatório ${ }^{25}$.

Como limitação do estudo observou-se o tamanho da amostra, já que o protocolo proposto demandava que os pacientes pudessem ser livremente mobilizados no leito, sabe-se que o doente crítico apresenta grande instabilidade e necessidade de manipulação mínima no período inicial do internamento. Todavia, os resultados alcançados foram interessantes, desta forma, sugere-se a necessidade de mais pesquisas, controladas e ensaios clínicos que abordem os efeitos da terapia manual na mecânica respiratória do paciente crítico.

O impacto clínico deste estudo se deve ao fato de que terapias complementares, como o protocolo proposto neste estudo, sejam empregadas a fim de proporcionar uma visão ampla sobre o desempenho do sistema respiratório de pacientes sob VM, considerando a estrutura óssea e muscular da caixa torácica, e podendo influenciar positivamente na função pulmonar e no desmame ventilatório.

\section{CONCLUSÃO}

Concluiu-se que o protocolo de terapia manual proposto apresentou efeitos positivos nas variáveis analisadas, Cdin e Pimáx, de pacientes críticos ventilados em modo espontâneo.

\section{FONTE DE FINANCIAMENTO}

Nada a declarar.

\section{CONFLITO DE INTERESSES}

Nada a declarar.

\section{REFERÊNCIAS}

1. Borges DL, Arruda LA, Rosa TRP, Costa MAG, Baldez TEP, Silva GJP. Influência da atuação fisioterapêutica no processo de ventilação mecânica de pacientes admitidos em UTI no período noturno após cirurgia cardíaca não complicada. Fisioter Pesqui. 2016;23(2):129-35. http://dx.doi. org/10.1590/1809-2950/14133523022016.

2. Cordeiro AL, Lima ASS, Matos ICO, Oliveira LVB, Guimarães AR, Carvalho SO, et al. Análise do tempo de ventilação mecânica e internamento em pacientes submetidos a cirurgia cardíaca. ABCS Health Sciences. 2017;42(1):3-7. http://dx.doi.org/10.7322/abcshs.v42i1.942.

3. França EÉ, Ferrari F, Fernandes P, Cavalcanti R, Duarte A, Martinez BP, et al. Physical therapy in critically ill adult patients: recommendations from the Brazilian Association of Intensive Care Medicine Department of Physical Therapy. Rev Bras Ter Intensiva. 2012 Mar;24(1):6-22. PMid:23917708.

4. Araújo JP No, Crespo AS, Araújo ML. Ventilação mecânica: alterações fisiológicas, indicações e parâmetros de ajuste. Rev Bras Anestesiol. 1996;46(3):187-98.

5. Faustino EA. Mecânica pulmonar de pacientes em suporte ventilatório na unidade de terapia intensiva. Conceitos e monitorização. Rev Bras Ter Intensiva. 2007;19(2):161-9. http://dx.doi.org/10.1590/S0103-507X2007000200004. PMid:25310774.

6. Pérez M, Mancebo J. Monitorización de la mecánica ventilatoria. Med Intensiva. 2006 Dez;30(9):4408. http://dx.doi.org/10.1016/S0210-5691(06)74567-3. PMid:17194401.

7. Condessa RL, Brauner JS, Saul AL, Baptista M, Silva ACT, Vieira SRR. Inspiratory muscle training did not accelerate weaning from mechanical ventilation but did improve tidal volume and maximal respiratory pressures: a randomised trial. J Physiother. 2013;59(2):101-7. http://dx.doi.org/10.1016/ S1836-9553(13)70162-0. PMid:23663795.

8. Ruppenthal JB, Groisman S, Azevedo M, Moura M, Hoff FC, Lima MP. Técnicas de terapia manual torácica através do método Reequilíbrio-Tóraco-Abdominal (RTA) melhoram a ventilação pulmonar em pacientes ventilados mecanicamente. Ter Man. 2011;9(44):424-8.

9. Gonçalves A, Galrão A, Souza P. Efeitos da terapia manual associada à cinesioterapia no processo de cura em pacientes internados em unidade de terapia intensiva (UTI) [Trabalho de Conclusão de Pós-graduação]. Salvador (BA): Escola Bahiana de Medicina e Saúde Pública; 2011.

10. De Moraes TP, Matilde INE, Yamauchi LY. Efeitos do método reequilíbrio toraco abdominal e da técnica de vibrocompressão torácica na mecânica do sistema respiratório. ASSOBRAFIR Ciencia. 2014;5(3):23-4.

11. Noll DR, Shores JH, Gamber RG, Herron KM, SwiftJJr. Benefits of osteopathic manipulative treatment for hospitalized elderly patients with pneumonia. J Am Osteopath Assoc. 2000;100(12):776-82. PMid:11213665.

12. Noll DR, Degenhardt BF, Johnson JC. Multicenter osteopathic pneumonia study in the elderly: subgroup analysis on hospital length of stay, ventilator-dependent respiratory failure rate, and in-hospital mortality rate. J Am Osteopath Assoc. 2016;116(9):574-87. PMid:27571294.

13. Santos JJA, Santos MCA, Carli J, Rocha P, Previatti KEK. Influência das técnicas de terapia manual osteopática na função respiratória. Arq Ciênc Saúde UNIPAR. 2015;19(13):191-7.

14. François R. Tratamento osteopático da caixa torácica. São Paulo: Andreoli; 2009.

15. Marek SM, Cramer JT, Fincher AL, Massey LL, Dangelmaier SM, Purkayastha S, et al. Acute effects of static and proprioceptive neuromuscular facilitation stretching on muscle strength and power output. J Athl Train. 2005;40(2):94-103. PMid:15970955. 
16. Coutinho EL, Gomes ARS, França CN, Oishi J, Salvini TF. Effect of passive stretching on the immobilized soleus muscle fiber morfology. Braz J Med Biol Res. 2004;37(12):1853-61. http://dx.doi.org/10.1590/S0100-879X2004001200011. PMid:15558192.

17. Teodori RM, Moreno MA, Fiore JF Jr, Oliveira ACS. Alongamento da musculatura inspiratória por intermédio da reeducação postural global (RPG). Rev. Bras. Fisioter. 2003;7(1):25-30.

18. Grinnan DC, Truwit JD. Clinical review: respiratory mechanics in spontaneous and assisted ventilation. Crit Care. 2005;9(5):472-84. http://dx.doi.org/10.1186/cc3516. PMid:16277736.

19. Lu Q, Rouby Jj. Measurement of pressure-volume curves in patients on mechanical ventilation: methods and significance. Crit Care. 2000;4(2):91-100. http://dx.doi. org/10.1186/cc662. PMid:11094498.

20. Novaes PA, Sanchez EGM, Sanchez HM. Medida das pressões respiratórias máximas em jovens saudáveis antes e após manobras de liberação diafragmática. Revista Inspirar Mov Saúde. 2013:5(2):1-5.

21. Rocha TDS. Eficácia da técnica manual de liberação diafragmática na distribuição regional de volume da caixa torácica, mobilidade do diafragma e função pulmonar de idosos saudáveis e com DPOC: um ensaio clínico. [dissertação]. Pernambuco (PE): Universidade Federal de Pernambuco; 2013.

22. Paulin E, Brunetto AF, Carvalho CRF. Efeitos de programa de exercícios físicos direcionado ao aumento da mobilidade torácica em pacientes portadores de doença pulmonar obstrutiva crônica. J Pneumol. 2003;29(5):287-94. http:// dx.doi.org/10.1590/S0102-35862003000500007.

23. Shah SB, Peters D, Jordan KA, Milner DJ, Fridén J, Capetanaki $Y$, et al. Sarcomere number regulation maintained after immobilization in desmin-null mouse skeletal muscle. J Exp Biol. 2001;204(Pt 10):1703-10. http://dx.doi.org/10.1242/ jeb.204.10.1703. PMid:11316490.

24. Moreno MA, Catai AM, Teodori RM, Borges BLA, Cesar MC, Silva E. Efeito de um programa de alongamento muscular pelo método de Reeducação Postural Global sobre a força muscular respiratória e a mobilidade toracoabdominal de homens jovens sedentários. J Bras Pneumol. 2007;33(6):67986. http://dx.doi.org/10.1590/S1806-37132007000600011. PMid:18200368.

25. Guimarães FS, Alves FF, Constantino SS, Dias CM, Menezes SLS. Avaliação da pressão inspiratória máxima em pacientes críticos não-cooperativos: comparação entre dois métodos. Braz J Phys Ther. 2007;11(3):233-8. http://dx.doi. org/10.1590/S1413-35552007000300010. 